\title{
Manuscript types
}

Public Health and Toxicology considers the following types of articles:

- Research Papers - reports of data from original research.

- Review Papers - comprehensive, authoritative, reviews within the journal's scope.

- Short Reports - brief reports of data from original research.

- Methodology Papers - Papers that present different methodological approaches that can be used to investigate problems in a relevant scientific field and to encourage innovation.

- Letters to the Editor - a response to authors of an original publication, or a very small article that may be relevant to readers.

- Editorials - articles written by members of the Editorial Board.

\section{Research Papers}

Articles reporting research may be full length or brief reports. These should report original research findings within the journal's scope. Papers should generally be a maximum of 4000 words in length, excluding a maximum of 5 tables, references, and abstract of the article, whilst it is recommended that the number of references should not exceed 36.

\section{Review Papers}

Comprehensive, authoritative, reviews within the journal's scope. There are two types of review papers:

- systematic review papers: respond to a specific research question, accrue from criterion-based selection of sources, include a quantitative synthesis and a statistical method (meta-analysis), and should adhere to PRISMA guidelines. Guidelines used for abstracting data and assessing data quality and validity should be noted in methods section.

- narrative review papers: the research question may be broad, and the scope of this review is to discuss a specific topic and keep the readers up-to-date about it. This type of review does not necessarily include a methodological approach and its synthesis is usually qualitative. Narrative reviews should include in a developments section, with details regarding data sources used, keywords applied, time restrictions and study types selected. Developments should be based on actual review articles.

All review papers should be generally less than 6000 words, excluding abstract, tables, figures and references. References should not exceed 50. Conclusion of the reviews should be specific and stem from the findings.

\section{Short Reports}

Brief reports of data from original research. Short reports are shorter versions of original articles, may include one table or figure, should not exceed 1500 words, and it is recommended that the number of references should not exceed 15. Short reports are suitable for the presentation of research that extends previously published research, including the reporting of additional controls and confirmatory results in other settings, as well as negative results. Authors must clearly acknowledge any work upon which they are building, both published and unpublished.

\section{Methodology Papers}

Methodology papers will present different methodological approaches that can be used to investigate problems in a relevant scientific field and to encourage innovation. It is suggested that case studies or practical examples, which can be existing ones, are included to demonstrate the consistency and applicability of the methodology.

Methodology papers should be generally less than 6000 words, excluding abstract, tables, figures and references. References should not exceed 50 .

\section{Letters to the editor}

A letter to the Editor is a brief report that is within the journal's scope and of particular interest to the community, but not suitable as a standard research paper. A maximum of ten articles may be included in the references. Letters to the Editor may be edited for clarity or length and may be subject to external peer review at the Editors' discretion. Letters intended for publication should be a maximum of 500 words, contain 10 references, and up to one table or figure. These rules apply both for research letters, and letters that respond to articles published in the journal. Letters to the editor are subject to editorial editing so as to streamline the article with the journal's style. Corrections to published articles are also published as a letter and linked to the corrected version of the article. 


\section{Editorials}

Editorials are written by members of the Editorial Board and may reflect current articles within Public Health and Toxicology or discuss significant national or international projects or initiatives.

\section{Manuscript formatting}

Public Health and Toxicology manuscripts are reviewed with the understanding that they:

- are original

- are not under consideration by any other publisher

- have not been previously published in whole or in part

- have not been previously accepted for publication

- will not be submitted elsewhere until a decision is reached regarding their publication

\section{General instructions}

The authors are encouraged to consult previous relevant publications in PHT to assist them in the preparation of the manuscript, especially the references and tables.

\section{Checklists}

We support the use of Checklists during manuscript preparation. Checklists are available for a number of study designs, including:

- randomized trials (CONSORT),

- systematic reviews (PRISMA),

- observational studies (STROBE),

- meta-analyses of observational studies (MOOSE) and

- qualitative studies (RATS).

\section{Text Formatting}

All manuscripts should be submitted in a Word format, they should be single column and 1.5 spaced. Margins should be one inch at the top, bottom and sides of the page. Font size should be 11-pt or 12-pt, standard font in 'Arial' or 'Times New Roman' typeface. Manuscripts should be formatted in full justified paragraphs and headings should be left-aligned. Maths should be editable text.

\section{Title Page}

The Title page should list the title of the article and suggestions for a short running title of no more than 60 characters (including spaces). Also include the authors names, affiliations and contact details including email address for the corresponding author. Affiliations should contain each author's department, institution (institute, university), city, country. The Title of the article should be clear, concise and highlighting the research topic. It should not include rhetorical questions, literary language, quotations and special symbols.

Authors cannot change the title of their article once it is accepted for publication, apart from very minor corrections.

\section{Abstract}

Authors are asked to supply a structured abstract of 250 words. For research articles, systematic reviews and brief reports, the abstract is limited to 250 words and should be structured as follows: Introduction, Methods, Results, and Conclusions. Abstracts for narrative reviews, study protocols and methodology papers are unstructured. Letters do not have an abstract.

Keywords

Include six keywords that describe your paper for indexing and for web searches of your manuscript.

\section{Main Text}

Research Papers, Systematic Review Papers and Short Reports sections are: Introduction, Methods, Results, Discussion, and Conclusions. Narrative Review Papers are not necessarily structured. It is suggested though to include the sections Introduction, Developments and Conclusion. Study Protocols consist of Introduction, Methods, Discussion, and Conclusions. Methodology Papers should consist of Introduction, Methodological approach, Case studies or Practical examples, Discussion, Conclusions. 
Use the guidelines below to structure these sections:

1. A short introduction which should end with the study's aims. The introduction should state clearly the objective of the paper as well as the context of the research or analysis.

2. A methods section which should describe the study design, setting, participants, measures, variables and statistical analysis performed. This section should also include information on the study's ethics procedures.

3. A results section, which should describe the study's main findings and important aspects within the tables.

4. A discussion section, which should include a discussion of the study's main findings, comparisons with other studies, potential policy implications, the study's strengths and limitations.

5. A conclusions section, which should be short, concise and based on the results of the current study. General conclusions that do not stem from the manuscript's results should be avoided.

\section{Declaration of Interests}

Declare any competing interests for each author. Public Health and Toxicology adheres to the International Committee of Medical Journal Editors (ICMJE) Recommendations for the Conduct, Reporting, Editing and Publication of Scholarly Work in Medical Journals. The author names on the ICMJE forms should be identical to the names in the manuscript.

\section{Funding}

All sources of funding for the research reported should be declared. The role of the funding body in the design of the study and collection, analysis, and interpretation of data and in writing the manuscript should be declared. The sentence should begin: "This work was supported by". Proposal numbers should be complete and accurate and provided in parentheses as follows: "(proposal number xxxx)"

\section{Acknowledgements}

This section is for acknowledging individuals and institutions whose support the authors wish to mention (it is not compulsory). Please acknowledge anyone who contributed towards the article who does not meet the criteria for authorship including anyone who provided professional writing services or materials. The Acknowledgements section should be kept to a minimum.

\section{Authors' contributions}

The individual contributions of authors to the manuscript should be specified in this section. Guidance and criteria for authorship can be found in our editorial policies. The authors have the option to give a brief outline of their contribution. Please use initials to refer to each author's contribution in this section.

Group authorship (for manuscripts involving a collaboration group): If you would like the names of the individual members of a collaboration Group to be searchable through their individual PubMed records, please ensure that the title of the collaboration Group is included on the title page and in the submission system and also include collaborating author names as the last paragraph of the "Acknowledgements" section. Please add authors in the format First Name, Middle initial(s) (optional), Last Name. You can add institution or country information for each author if you wish, but this should be consistent across all authors.

\section{Tables, Figures and Supplementary material}

Tables and Figures should be placed at the end of the manuscript and be numbered sequentially in order of appearance in the text. However authors should ensure that every table or figure is referred to in the body of the text. Each table or figure should be accompanied by a short, descriptive title (including the place, year and sample size) and in the footnote should define any acronyms, abbreviations or symbols used, statistical methods applied and any other information needed so that the table or figure may stand alone in the HTML and pdf version of the manuscript. Superscripts used to refer to table footnotes should be lowercase alphabetical symbols. Captions should be given separately above the tables or figures. Numbers in the table should not contain commas, and numbers less than unity should have a zero in front of the decimal point. Decimal numbers should be represented with the use of a full stop.

The number of actual tables (no sub-tables) that an article can contain should not exceed five. However, they have to be of value as determined by peer review. Extra tables can be included in the Supplementary material. The content of the tables should be such that the data are of sufficient resolution for comfortable reading. Tables should be submitted in their original Word format (not via Excel), and they should be legible. Avoid using vertical rules. Horizontal rules should be used only above and below column headings and at the bottom of the table. Do not create a table using only tabs or spaces to create columns. Tables should not duplicate material contained in the main text.

The number of figures should not exceed four. Extra figures can be included in the Appendix. Figures can be submitted in 
greyscale, black or white or in color as the journal is published online. The journal prefers that figures be created in Excel. Do not embed a figure file as a picture into Excel or Word, but submit them as individual files. The data of figures should be attached in a Word format. Size the figure to the column or page width of the journal, and set the resolution at 300 dpi or greater. Figures containing a large amount of text, particularly flow diagrams, should be send in an editable form.

Supplementary material should be submitted in a Word format.

\section{References}

These must be numbered sequentially, as they first appear in the text. They should be within square brackets without spaces before or afterwards. Where more than one reference is cited, these should be separated by a comma, for example, [1, 12, 16], while for a continuous sequence of numbers, give the first and last number of the sequence separated by a hyphen, for example, [4-7]. The Reference list should thus be sequentially numbered using plain text (i.e. without the use of footnote or endnote etc). Please check the reference list and ensure that there is no duplication of references.

\section{Article citations}

These follow the AMA format.

\section{Journal articles in Print}

Citation format:

Authors. Title. Journal name. Year;Volume number(issue number):initial-final page.

(Note: Use et al. instead of names after the 6th author)

Example:

Kjellstrom Tord, Lemke Bruno, Otto Matthias. Climate conditions, workplace heat and occupational health in South-East Asia in the context of climate change. WHO South East Asia J Public Health. 2017;6(2):69-73.

\section{Journal articles Online}

All references that are from journal articles must contain a Digital Object Identifier (DOI): A DOI is a unique character string created to identify a digital object, such as a journal article in an online environment. The DOI is a permanent identifier of all versions of an article and the DOI for a document remains fixed over the lifetime of the document. The DOI must be included in the citation.

Citation format:

Authors. Title. Journal name. Year;Volume number(issue number):initial-final page. Doi.

Example:

Ramón Escuriet, Laura García-Lausin, Isabel Salgado-Poveda, Rocio Casañas, Gemma Robleda, Olga Canet, Mercedes PérezBotella et al. Midwives' contribution to normal childbirth care: Cross-sectional study in public health settings, the MidconBirth Study protocol. Eur J Midwifery 2017;1(September):4. doi:10.18332/ejm/76820

\section{Books}

Citation format:

Authors. Title. Volume number (for more than 1 volume). Edition number. Place of publication: Name of publisher; year of publication:page numbers.

(Note: Use et al. instead of names after the 6th author)

Example:

Patterson James T. The dread disease: cancer and modern American culture.

Cambridge, MA: Harvard University Press; 1989.

\section{Web links and URLs}

All web links and URLs, including links to the authors' own websites, should be given a reference number, and included in the reference list, rather than within the text of the manuscript. They should be provided in full, including both the title of the item, the title of the site and the URL, as well as the date the site was accessed.

\section{Website}

Citation format:

Authors. Title. Name of the website. URL. Published date. Updated date. Accessed date.

Example:

The OASI care Bundle Project. https://www.rcog.org.uk/OASICareBundle. Accessed September 2017. 


\section{Report Online}

Citation format:

Authors or Institution. Title. URL. Published date. Accessed date.

Example:

World Health Organization. Equitable access to essential medicines: a framework for collective action. http://apps.who.int/ medicinedocs/pdf/s4962e/s4962e.pdf. Published March, 2004. Accessed November 24, 2017.

\section{How to submit}

\section{Online Submission}

Public Health and Toxicology uses the online system "Editorial System" for manuscript submission, peer review and editorial handling. Submissions received by e-mail will not be considered. The journal does not have submission fees. Within the submission system the corresponding author will have the ability to upload a cover letter and will also be able to select pre-identified statements related to the originality of the work, the potential conflicts of interest, authorship and the role of funding sources in manuscript preparation. Additional information may be requested by the Editorial Board so as to ensure the integrity and ethics of the manuscript.

\section{Article Processing Charges}

Public Health and Toxicology does not have any Article Processing Charges.

\section{Preprints}

We encourage authors to submit manuscripts that have been previously published as pre-prints. Authors publishing in our journals may have shared their work ahead of submission, as well as during the journal's peer review process on repositories or preprint servers (such as MedarXiv, PeerJ Preprints etc), as long as the server does not impose restrictions upon the author's full copyright and re-use rights. Preprints are not considered prior publication, but it is essential that the preprint submitted in our journals is not under consideration by any other journal. In order to make sure that correct attribution is given to the original source (either it is repositories or preprint servers), we suggest that the preprint is listed within the acknowledgement section of the manuscript.

If the manuscript is accepted for publication, authors are encouraged to link their preprint with the published article, in order to enable their readers to access the peer-reviewed version. Preprints that undergo a peer review process through Public Health and Toxicology will be published under the same license used for all the articles in the journal (CC-BY-NC 4.0). 